\title{
HYPERCONTRACTIVITY IN FINITE-DIMENSIONAL MATRIX ALGEBRAS
}

\author{
MARIUS JUNGE, CARLOS PALAZUELOS \\ JAVIER PARCET, MATHILDE PERRIN
}

\begin{abstract}
We obtain hypercontrativity estimates for a large class of semigroups defined on finite-dimensional matrix algebras $\mathbb{M}_{n}$. These semigroups arise from Poisson-like length functions $\psi$ on $\mathbb{Z}_{n} \times \mathbb{Z}_{n}$ and provide new hypercontractive families of quantum channels when $\psi$ is conditionally negative. We also study the optimality of our estimates.
\end{abstract}

\section{Introduction}

Hypercontractivity is a phenomenon which arises naturally in different subjects. Originally, it was discovered in the early 70's simultaneously in harmonic analysis and quantum field theory. Since then, hypercontractivity has found connections with many different areas of mathematics, we refer to Gross' excellent survey [15]. More recently, hypercontractivity estimates appear to be of great interest in topics like complexity theory, classical and quantum information and condense matter theory. We refer for instance to $[5,18,19,20,23]$ and the references therein.

Two fundamental results in the theory play the role of basic building blocks for other more elaborated hypercontractivity estimates. The first one is the so-called 'two-point inequality' originally proved by Bonami [10] and independently by Gross [14]. This result can be considered as the birth of the theory. Given $1<p \leq q<\infty$, it gives the optimal time above which the Poisson semigroup on $\mathbb{Z}_{2}$ is contractive from $L_{p}\left(\mathbb{Z}_{2}\right)$ to $L_{q}\left(\mathbb{Z}_{2}\right)$. From an operator algebra viewpoint, it can be regarded as the Ornstein-Uhlenbeck semigroup on the Clifford algebra with one generator. Bonami's inequality was essential for Beckner's famous theorem on the optimal Hausdorff-Young inequality [6]. Other applications to information theory are described in [15]. The second result is an optimal convexity inequality for matrices by BallCarlen-Lieb in [4]. This was crucial to find the optimal hypercontractivity estimates for the Ornstei n-Uhlenbeck semigroup defined on the fermonic Clifford algebra, a long standing problem finally solved by Carlen-Lieb in [11]. In conjunction with central limit-type of theorems, it has also been a key tool to find several generalizations of Carlen-Lieb results [9, 17]. 
Carlen and Lieb followed in [11] an inductive argument due to the tensor product structure of the fermonic Clifford algebra, which can be represented in the algebras $\mathbb{M}_{2^{n}}=\bigotimes_{j=1}^{n} \mathbb{M}_{2}$. In fact, most applications of the two-point inequality and of Ball-Carlen-Lieb inequality are precisely based on iteration arguments by finding a suitable tensor product structure in the problem. This excludes very basic scenarios like the cyclic groups $\mathbb{Z}_{n}$ or the matrix algebras $\mathbb{M}_{n}$ when $n$ is not a power of 2 .

The aim of the present work is to transfer some recent results on the cyclic groups $[16,26]$ to obtain hypercontractivity estimates for certain semigroups defined on finite-dimensional matrix algebras $\mathbb{M}_{n}$. More precisely, given $n \geq 1$, consider the $(n \times n)$-unitary matrices

$$
u_{k}=\sum_{j=1}^{n} e^{\frac{2 \pi i k j}{n}} e_{j, j} \quad \text { and } \quad v_{\ell}=\sum_{j=1}^{n} e_{j, \overline{j+\ell}}
$$

for any $0 \leq k, \ell \leq n-1$. Here $\overline{j+\ell}$ stands for $j+\ell \bmod n$ and $e_{i, j}$ denotes the matrix with all entries equal zero up to the entry $(i, j)$, which equals one. The key objects in the sequel will be the unitary matrices

$$
w_{k, \ell}=u_{k} v_{\ell} \quad \text { for any } \quad 0 \leq k, \ell \leq n-1,
$$

which form an orthonormal basis of $\mathbb{M}_{n}$ (see Lemma 1.1). Let us denote by $\mathrm{G}=\mathbb{Z}_{n} \times \mathbb{Z}_{n}$ the cartesian product of the cyclic group $\mathbb{Z}_{n}$ with itself. Given any function $\psi: \mathbb{Z}_{n} \times \mathbb{Z}_{n} \rightarrow \mathbb{R}_{+}$, we will say that $\psi$ is a Poisson-like length function whenever the following conditions hold

- Spectral gap: $\sigma:=\min _{g \neq e} \psi(g)>0$,

- Subadditivity: $\psi\left(g+g^{\prime}\right) \leq \psi(g)+\psi\left(g^{\prime}\right)$,

- Length conditions: $\psi(e)=0, \psi(g)=\psi(-g)$.

If in addition $\psi$ is conditionally negative

$$
\sum_{g \in \mathrm{G}} a_{g}=0 \Rightarrow \sum_{g, h \in \mathrm{G}} \overline{a_{g}} a_{h} \psi(h-g) \leq 0,
$$

then we say that $\psi$ is a conditionally negative Poisson-like length function or c.n.P. length in short. We should warn the reader that our definition of Poisson-like length in [16] already considered the conditionally negativity and the so called exponential growth property. Given a Poisson-like length $\psi$, consider the semigroup $\mathcal{S}_{\psi, t}: \mathbb{M}_{n} \rightarrow \mathbb{M}_{n}$ determined by

$$
\mathcal{S}_{\psi, t}\left(w_{k, \ell}\right)=e^{-t \psi(k, \ell)} w_{k, \ell} \quad \text { for any } t \geq 0 .
$$

Let $L_{p}^{n}$ denote the noncommutative $L_{p}$-space on $\mathbb{M}_{n}$ with respect to the normalized trace $\operatorname{tr}_{n}$. In other words, $L_{p}^{n}$ is just $\mathbb{M}_{n}$ equipped with the norm

$$
\|A\|_{L_{p}^{n}}=\frac{1}{n^{1 / p}} \operatorname{tr}\left(|A|^{p}\right)^{1 / p}
$$

Our main result provides the following hypercontractivity estimates for $\mathcal{S}_{\psi, t}$. 
Theorem A. Let

$$
\psi: \mathbb{Z}_{n} \times \mathbb{Z}_{n} \rightarrow \mathbb{R}_{+}
$$

be a Poisson-like length satisfying the growth condition

$$
N_{R}(\psi):=\left|\left\{(k, \ell) \in \mathbb{Z}_{n} \times \mathbb{Z}_{n}: \psi(k, \ell) \leq R\right\}\right| \leq C \rho^{R}
$$

for any $R \geq 0$ and certain $C, \rho>0$. Then, the following holds:

(i) For any even integer $q$ we have

$$
\left\|\mathcal{S}_{\psi, t}: L_{2}^{n} \rightarrow L_{q}^{n}\right\|=1 \quad \text { for all } \quad t \geq t_{1}(q, C, \rho, \sigma),
$$

where $t_{1}=\max \left\{\frac{1}{\sigma}\left(\left(\frac{1}{2}-\frac{1}{q}\right) \log \left(2 C \rho^{\sigma}\right)+\frac{1}{2} \log (q-1)\right), \log \rho\right\}$.

(ii) If $\psi$ is also conditionally negative and $1<p \leq q<\infty$

$$
\begin{gathered}
\left\|\mathcal{S}_{\psi, t}: L_{p}^{n} \rightarrow L_{q}^{n}\right\|=1 \quad \text { for all } \quad t \geq t_{2}(p, q, C, \rho, \sigma), \\
\text { where } t_{2}=\max \left\{\frac{1}{\sigma}\left(\frac{1}{4} \log \left(2 C \rho^{\sigma}\right)+\frac{1}{2} \log 3\right), \log \rho\right\} \log \left(\frac{q-1}{p-1}\right) .
\end{gathered}
$$

Remarkably, the unitaries $w_{k, \ell}$ are very well-known in different contexts of quantum information and computation like quantum codes [8, 13, 22], quantum channel theory $[1,2]$ and so on. Note that in the case $k=\ell=1$ we are dealing essentially with the Pauli matrices. An explicit formulation of Theorem A (ii) in terms of these unitaries is

$$
n^{\frac{1}{p}-\frac{1}{q}}\left\|\sum_{k, \ell=0}^{n-1} a_{k, \ell} e^{-t \psi(k, \ell)} w_{k, \ell}\right\|_{S_{q}^{n}} \leq\left\|\sum_{k, \ell=0}^{n-1} a_{k, \ell} w_{k, \ell}\right\|_{S_{p}^{n}}
$$

for any $a_{k, \ell} \in \mathbb{C}$ and $t \geq t_{2}(p, q, C, \rho, \sigma)$. According to Schoenberg's theorem $\mathcal{S}_{\psi, t}$ is a unital, normal, completely positive and trace preserving map when $\psi$ is conditionally negative. Therefore, in this situation our semigroups are formed by doubly-stochastic quantum channels (completely positive, unital and trace preserving maps). Since hypercontractivity has been shown to be a very useful tool to find bounds on the convergence time of semigroups of quantum channels $[12,18,19]$, our results could be of interest in quantum information theory. Actually, similar results have been already studied for semigroups consisting of tensor products of $q$-bits channels, i.e. of the form $e^{-t_{1} H_{n}} \otimes \cdots \otimes e^{-t_{n} H_{n}}$ acting on $\mathbb{M}_{2^{n}}$, where the tensor product structure was crucially exploited by the authors, see for instance $[21,24]$.

The main novelties of Theorem A are three. The first one is clearly the fact that we consider general matrix algebras $\mathbb{M}_{n}$, lacking a tensor product structure. The second one is the generality of our semigroups, defined by means of very few restrictions on the generating function $\psi$. As we shall explain, our conditions are either necessary for hypercontractivity or very natural. The third one is that the time $t$ provided in Theorem A is optimal in different senses. More precisely, when $q>>\max \{C, \rho\}$ we obtain a time of order $\frac{1}{2 \sigma} \log (q-1)$, which is optimal. Moreover, when $\rho>>\max \{q, C\}$ we get a time of order $\log \rho$, which cannot be improved. 
The key point to prove Theorem A is that the combinatorics involved in the problem are exactly the same as those appearing when one studies the Poisson semigroup on $\mathbb{Z}_{n} \times \mathbb{Z}_{n}$ associated to a function $\psi$, for which some hypercontractivity results are obtained in $[16,26]$. That is, even when no group von Neumann algebra is described by the matrix algebra $\mathbb{M}_{n}$, the results developed in [26] can be transfered to the matrix algebra setting whenever we consider $q$ an even integer.

The price for a general statement like Theorem A is that the result might be not optimal for concrete choices of lengths. It is important to point out that for such lengths, one may use transference from a more sophisticated combinatorial approach in [16]. As an example which is particularly relevant in harmonic analysis, we will treat here the case of the standard conditionally negative Poisson-like length function on $\mathbb{Z}_{n} \times \mathbb{Z}_{n}$ defined by the metric of the Cayley graph

$$
|(k, \ell)|=\min \{k, n-k\}+\min \{\ell, n-\ell\} .
$$

In the following result we find the optimal hypercontractivity time.

Theorem B. Given $n \geq 4$

$$
\left\|\mathcal{S}_{||, t}: L_{2}^{n} \rightarrow L_{q}^{n}\right\|=1 \Leftrightarrow t \geq \frac{1}{2} \log (q-1)
$$

whenever one of the following conditions hold

(i) $q \in 2 \mathbb{Z}_{+}$and $n$ is even or $n=5$,

(ii) $q \in 2 \mathbb{Z}_{+}$and $n \geq 7$ is odd with $n \geq q$.

In particular, for any $1<p \leq q<\infty$ and $n \geq 4$ we find that

$$
\left\|\mathcal{S}_{\psi, t}: L_{p}^{n} \rightarrow L_{q}^{n}\right\|=1 \quad \text { for all } \quad t \geq \frac{\log 3}{2} \log \left(\frac{q-1}{p-1}\right) .
$$

As pointed in [16], one could follow a similar approach to deal with other functions $\psi$ and groups. However, the dependance on certain computational calculus forces that every proof must be done independently. We refer to [16] for an explanation of the general method which could be used to generalize Theorem B above to other concrete lengths which might be of interest.

\section{Proof of Theorem A}

In this section, we shall prove Theorem A and analyze its optimality in various aspects. We begin with a collection of properties of the unitary matrices $w_{k, \ell}$ introduced above. The proof is trivial.

Lemma 1.1. Given $0 \leq k, \ell \leq n-1$, we have:

(i) $u_{k} u_{\ell}=u_{\ell} u_{k}=u_{k+\ell}$ and $v_{k} v_{\ell}=v_{\ell} v_{k}=v_{k+\ell}$.

(ii) $u_{k} v_{\ell}=e^{-\frac{2 \pi i k \ell}{n}} v_{\ell} u_{k}$ and $\operatorname{tr}_{n}\left(w_{k, \ell}\right)=\delta_{(k, \ell)=(0,0)}$. 
(iii) $\left(w_{k, \ell}\right)_{k, \ell=1}^{n}$ is a Hilbert-Schmidt orthonormal basis of $\mathbb{M}_{n}$ with respect to the inner product $\langle u, v\rangle=\operatorname{tr}_{n}\left(u v^{*}\right)$.

Let us recall some basic notions of harmonic analysis on the finite abelian group $\mathrm{G}=\mathbb{Z}_{n} \times \mathbb{Z}_{n}$. Let us equip $\mathrm{G}$ with the counting measure, so that its Pontryagin dual $\widehat{G}=G$ is endowed with the normalized measure. Then the family

$$
\widetilde{w}_{k, \ell}=e^{\frac{2 \pi i\langle(k, \ell), \cdot\rangle}{n}} \quad \text { for } \quad 0 \leq k, \ell \leq n-1
$$

forms an orthonormal basis of $\ell_{2}(\widehat{\mathrm{G}})$. Moreover, it satisfies the analog of Lemma 1.1 (iii). Namely, we have $\operatorname{tr}_{\mathrm{G}}\left(\widetilde{w}_{k, \ell}\right)=\delta_{(k, \ell)=(0,0)}$, where the trace is given by $\operatorname{tr}_{\mathrm{G}}(f)=\frac{1}{n^{2}} \sum_{(a, b) \in \widehat{\mathrm{G}}} f(a, b)$. Given $f: \widehat{\mathrm{G}} \rightarrow \mathbb{C}$, we may decompose

$$
f=\sum_{0 \leq k, \ell \leq n-1} \widehat{f}(k, \ell) \widetilde{w}_{k, \ell}
$$

where the $(k, \ell)$-th Fourier coefficient of $f$ is given by

$$
\widehat{f}(k, \ell)=\frac{1}{n^{2}} \sum_{(a, b) \in \widehat{\mathrm{G}}} f(a, b) e^{-\frac{2 \pi i\langle(k, \ell),(a, b)\rangle}{n}} .
$$

For a Poisson-like length $\psi: \mathrm{G} \rightarrow \mathbb{R}_{+}$its Poisson semigroup $\mathcal{P}_{\psi, t}$ is given by

$$
\mathcal{P}_{\psi, t}\left(\widetilde{w}_{k, \ell}\right)=e^{-t \psi(k, \ell)} \widetilde{w}_{k, \ell} .
$$

This semigroup is known to satisfy some hypercontractivity properties. More precisely, it is established in [26, Proposition 13] and in [16, Theorem B] that for any Poisson-like length function $\psi$ with order of growth given by $N_{R}(\psi) \leq C \rho^{R}$ (recall the definition of $N_{R}(\psi)$ in the statement of Theorem A) and any $2 \leq q<\infty$ we have

$$
\left\|\mathcal{P}_{\psi, t}: L_{2}\left(\mathbb{Z}_{n} \times \mathbb{Z}_{n}\right) \rightarrow L_{q}\left(\mathbb{Z}_{n} \times \mathbb{Z}_{n}\right)\right\|=1 \quad \text { for all } \quad t \geq t_{1}(q, C, \rho, \sigma)
$$

for some time $t_{1}(q, C, \rho, \sigma)$. Ricard and Xu's result [26] yields a better time

$$
t(q, C, \rho, \sigma)=\max \left\{\frac{1}{\sigma}\left(\left(\frac{1}{2}-\frac{1}{q}\right) \log \left(2 C \rho^{\sigma}\right)+\frac{1}{2} \log (q-1)\right), \log \rho\right\} .
$$

Their estimate relies on a deep result, an extension of the Ball-Carlen-Lieb convexity inequality to any semifinite von Neumann algebra. Note that they proved it in the normalized situation $\sigma=1$, but we can deduce the time $t_{1}(q, C, \rho, \sigma)$ for any spectral gap $\sigma$ by keeping track of the parameter $\sigma$ in their proof. In contrast, the arguments in [16] are much more elementary and the proof of Theorem B there is rather simple and relies entirely on a combinatorial approach.

Proof of Theorem A. Our transference argument relies on the fact that $\mathcal{P}_{\psi, t}$ and $\mathcal{S}_{\psi, t}$ act similarly on the orthonormal basis $\left(\widetilde{w}_{k, \ell}\right)_{0 \leq k, \ell \leq n-1}$ and $\left(w_{k, \ell}\right)_{0 \leq k, \ell \leq n-1}$ respectively, which share the property that its trace is zero except when $(k, \ell)=(0,0)$. The lack of commutativity of the $w_{k, \ell}$ 's yields an additional (unimodular) phase when computing the $L_{q}$-norm for an even 
integer $q$, which can be killed after a triangular inequality thanks to the unconditionality of the $L_{2}$-norm. We claim that for $q \in 2 \mathbb{Z}_{+}$

$$
\left\|\mathcal{S}_{\psi, t}: L_{2}^{n} \rightarrow L_{q}^{n}\right\| \leq\left\|\mathcal{P}_{\psi, t}: L_{2}\left(\mathbb{Z}_{n} \times \mathbb{Z}_{n}\right) \rightarrow L_{q}\left(\mathbb{Z}_{n} \times \mathbb{Z}_{n}\right)\right\|
$$

Thus Theorem A (i) will follow by (1.1). Indeed, we will prove that for any even integer $q$ and any matrix $A=\sum_{k, \ell=0}^{n-1} a_{k, \ell} w_{k, \ell} \in \mathbb{M}_{n}$, the following estimate holds

$$
\left\|\mathcal{S}_{\psi, t}(A)\right\|_{q} \leq\left\|\mathcal{P}_{\psi, t}(f)\right\|_{q} \leq\left\|\mathcal{P}_{\psi, t}\right\|_{2 \rightarrow q}\|f\|_{2}=\left\|\mathcal{P}_{\psi, t}\right\|_{2 \rightarrow q}\|A\|_{2},
$$

where

$$
f=\sum_{k, \ell=0}^{n-1}\left|a_{k, \ell}\right| \widetilde{w}_{k, \ell} \in L_{\infty}\left(\mathbb{Z}_{n} \times \mathbb{Z}_{n}\right) .
$$

The last identity of (1.3) is clear by unconditionality of the $L_{2}$-norm. Thus it suffices to justify the first inequality. For any even integer $q$, we may write $\left\|\mathcal{S}_{\psi, t}(A)\right\|_{L_{q}^{n}}^{q}$ as follows

$$
\sum_{\substack{\left(k_{j}, \ell_{j}\right) \in \mathbb{Z}_{n} \times \mathbb{Z}_{n} \\ 1 \leq j \leq q}} \overline{a_{k_{1}, \ell_{1}}} a_{k_{2}, \ell_{2}} \cdots a_{k_{q}, \ell_{q}} e^{-t \sum_{j} \psi\left(k_{j}, \ell_{j}\right)} \operatorname{tr}_{n}\left(w_{k_{1}, \ell_{1}}^{*} w_{k_{2}, \ell_{2}} \cdots w_{k_{q}, \ell_{q}}^{*}\right) \text {. }
$$

According to Lemma 1.1 (ii), this can be written as

$$
\sum_{\begin{array}{c}
\left(k_{j}, \ell_{j}\right) \in \mathbb{Z}_{n} \times \mathbb{Z}_{n} \\
-k_{1}+k_{2}-\cdots+k_{q}=0 \\
-\ell_{1}+\ell_{2}-\cdots+\ell_{q}=0
\end{array}} \bar{a}_{k_{1}, \ell_{1}} a_{k_{2}, \ell_{2}} \cdots a_{k_{q}, \ell_{q}} e^{-t \sum_{j} \psi\left(k_{j}, \ell_{j}\right)} \alpha\left(k_{1}, \ell_{1}, \cdots, k_{q}, \ell_{q}\right)
$$

where $\alpha\left(k_{1}, \ell_{1}, \cdots, k_{q}, \ell_{q}\right)$ is a complex number of modulus one coming from the commutation $r$ elations given in Lemma 1.1 (ii). Thus, by the triangular inequality we get

$$
\left\|\mathcal{S}_{\psi, t}(A)\right\|_{L_{q}^{n}}^{q} \leq \sum_{\substack{\left(k_{j}, \ell_{j}\right) \in \mathbb{Z}_{n} \times \mathbb{Z}_{n} \\-k_{1}+k_{2}-\cdots+k_{q}=0 \\-\ell_{1}+\ell_{2}-\cdots+\ell_{q}=0}}\left|a_{k_{1}, \ell_{1}} a_{k_{2}, \ell_{2}} \cdots a_{k_{q}, \ell_{q}}\right| e^{-t \sum_{j} \psi\left(k_{j}, \ell_{j}\right)} .
$$

Observe that similar computations replacing the matrix unitary $w_{k, \ell}$ by the commutative unitary $\widetilde{w}_{k, \ell}$ yield that the right hand side above is equal to $\left\|\mathcal{P}_{\psi, t}(f)\right\|_{L_{q}\left(\mathbb{Z}_{n} \times \mathbb{Z}_{n}\right)}$. This proves (1.3) and ends the proof of Theorem A (i).

If $\psi$ is in addition conditionally negative, $\mathcal{S}_{\psi, t}$ is a unital cp-map by Schoenberg's theorem and it has positive maximizers, see [16, Lemma 1.1] for details. In that case, we may combine Gross' extrapolation technique with interpolation results to deduce $L_{p} \rightarrow L_{q}$ hypercontractivity estimates from $L_{2} \rightarrow L_{4}$ ones. Namely, if we have $\left\|\mathcal{S}_{\psi, t}: L_{2}^{n} \rightarrow L_{4}^{n}\right\|=1$ for all $t \geq t_{2,4}$ then we claim that for any $1<p \leq q<\infty$ we have $\left\|\mathcal{S}_{\psi, t}: L_{p}^{n} \rightarrow L_{q}^{n}\right\|=1$ for all $t \geq t_{2,4} \log (q-1 / p-1)$. We refer to [16, Appendix $\left.\mathrm{A}\right]$ for a proof with the time $t_{2,4}=\frac{1}{2 \sigma} \log 3$, where we used the $L_{p}$-regularity of the associated Dirichlet form coming from [25] in the tracial case. Then our claim follows 
by adapting these arguments for an arbitrary time $t_{2,4}$. Applying it to the time $t_{2,4}=t_{1}(4, C, \rho, \sigma)$ obtained in Theorem A (i) yields assertion (ii).

Remark 1.2. We claim that

$$
\left\|\mathcal{S}_{\psi, t}: L_{2}^{n} \rightarrow L_{q}^{n}\right\|=1 \Rightarrow t \geq \max \left\{\frac{1}{2 \sigma} \log (q-1), \frac{1}{\max \psi}\left(\frac{q-2}{2 q}\right) \log n\right\} .
$$

This proves certain optimality in the order of our estimates:

a) When $q$ is large compared to $\rho$ and $C$, the hypercontractivity time in Theorem A (i) behaves like $\frac{1}{2 \sigma} \log (q-1)$, which is optimal from the claim above.

b) When $\rho$ is large compared to $q$ and $C$ the time we find in Theorem A (i) behaves like $\log \rho$, which (especially for $\sigma \sim 1$ ) coincides with the order of our lower estimate above. Indeed, for $C=1$ for instance, we have $\rho^{\sigma} \leq n^{2}$ and obtain that $t$ must be greater than or equal to $\frac{\sigma}{\max \psi}\left(\frac{q-2}{4 q}\right) \log \rho$. It is easy to see that by letting $\rho=n^{2 / \sigma}$ we get $N_{R}(\psi) \leq n^{2}=\rho^{\sigma} \leq \rho^{R}$ since $R \geq \sigma$.

Let us justify our claim. To prove the first lower estimate $t \geq \frac{1}{2 \sigma} \log (q-1)$ we may use a well-known argument which in fact works for arbitrary indices $1<p \leq q<\infty$

$$
\left\|\mathcal{S}_{\psi, t}: L_{p}^{n} \rightarrow L_{q}^{n}\right\|=1 \Rightarrow t \geq \frac{1}{2 \sigma} \log \left(\frac{q-1}{p-1}\right) .
$$

To see this, pick $(k, \ell) \in \mathbb{Z}_{n} \times \mathbb{Z}_{n}$ with $\psi(k, \ell)=\sigma$ and $\varepsilon>0$. Define

$$
A= \begin{cases}I+\varepsilon\left(w_{k, \ell}+w_{k, \ell}^{*}\right) & \text { if }(k, \ell) \neq\left(\frac{n}{2}, \frac{n}{2}\right) \\ I+\varepsilon w_{n / 2, n / 2} & \text { if } n \equiv 0 \bmod 4 \text { and }(k, \ell)=\left(\frac{n}{2}, \frac{n}{2}\right) \\ I+i \varepsilon w_{n / 2, n / 2} & \text { if } n \equiv 2 \bmod 4 \text { and }(k, \ell)=\left(\frac{n}{2}, \frac{n}{2}\right) .\end{cases}
$$

In the first case $\operatorname{tr}_{n}\left(w_{k, \ell}\right)=\operatorname{tr}_{n}\left(w_{k, \ell}^{2}\right)=\operatorname{tr}_{n}\left(\left(w_{k, \ell}^{*}\right)^{2}\right)=\operatorname{tr}_{n}\left(w_{k, \ell}^{*}\right)=0$ and

$$
\begin{aligned}
& \|A\|_{L_{p}^{n}}=\left[\operatorname{tr}_{n}\left(\left(I+2 \varepsilon\left(w_{k, \ell}+w_{k, \ell}^{*}\right)+\varepsilon^{2}\left(w_{k, \ell}^{2}+\left(w_{k, \ell}^{*}\right)^{2}+2 I\right)\right)^{\frac{p}{2}}\right)\right]^{\frac{1}{p}} \\
& =\left[\operatorname{tr}_{n}\left(I+p \varepsilon\left(w_{k, \ell}+w_{k, \ell}^{*}\right)+\frac{p}{2}(p-1) \varepsilon^{2}\left(w_{k, \ell}^{2}+\left(w_{k, \ell}^{*}\right)^{2}+2 I\right)+o\left(\varepsilon^{2}\right)\right)\right]^{\frac{1}{p}} \\
& =\left(1+p(p-1) \varepsilon^{2}+o\left(\varepsilon^{2}\right)\right)^{\frac{1}{p}}=1+(p-1) \varepsilon^{2}+o\left(\varepsilon^{2}\right) .
\end{aligned}
$$

The same computation yields

$$
\left\|\mathcal{S}_{\psi, t}(A)\right\|_{L_{q}^{n}}=1+(q-1) e^{-2 t \sigma} \varepsilon^{2}+o\left(\varepsilon^{2}\right) .
$$

Thus, by comparing these two norms we easily obtain (1.4). In the case $(k, \ell)=\left(\frac{n}{2}, \frac{n}{2}\right)$, since $w_{n / 2, n / 2}^{2}=(-1)^{n / 2} I$ the same argument gives rise to the identity

$$
\|A\|_{L_{p}^{n}}=1+\frac{1}{2}(p-1) \varepsilon^{2}+o\left(\varepsilon^{2}\right)
$$

and we obtain the same conclusion, which completes the proof of (1.4). 
Let us now prove the second lower estimate

$$
t \geq \frac{1}{\max \psi} \frac{q-2}{2 q} \log n .
$$

Assume that $\left\|\mathcal{S}_{\psi, t}\right\|_{2 \rightarrow q}=1$ and let $A=\sum_{k, \ell=0}^{n-1} a_{k, \ell} w_{k, \ell} \in \mathbb{M}_{n}$. Then, we may write $A_{t}=\sum_{k, \ell} a_{k, \ell} e^{t \psi(k, \ell)} w_{k, \ell}$ so that $S_{\psi, t} A_{t}=A$. This yields

$$
\|A\|_{L_{q}^{n}} \leq\left(\sum_{k, \ell=0}^{n-1}\left|a_{k, \ell} e^{t \psi(k, \ell)}\right|^{2}\right)^{1 / 2} \leq e^{t \max \psi}\|A\|_{L_{2}^{n}} .
$$

Since $\left\|i d: L_{2}^{n} \rightarrow L_{q}^{n}\right\|=n^{\frac{1}{2}-\frac{1}{q}}$, we deduce that $t$ must satisfy

$$
e^{t \max \psi} \geq n^{\frac{1}{2}-\frac{1}{q}} \Rightarrow t \geq \frac{1}{\max \psi}\left(\frac{1}{2}-\frac{1}{q}\right) \log n .
$$

Remark 1.3. Our conditions on the generating lengths $\psi: \mathbb{Z}_{n} \times \mathbb{Z}_{n} \rightarrow \mathbb{R}_{+}$ are not very restrictive. Namely, the existence of a spectral gap $\sigma>0$ is a necessary condition for hypercontractivity. On the other hand, the conditional negativity, subadditivity and symmetry of the length are very natural in this context. We refer to [16] for more detailed explanations.

\section{Proof of Theorem B}

In the proof of Theorem A we have seen that, to study the $L_{2}^{n} \rightarrow L_{q}^{n}$ hypercontractivity of $\mathcal{S}_{\psi, t}$ for $q$ an even integer, it suffices to study the hypercontractivity $L_{2}\left(\mathbb{Z}_{n} \times \mathbb{Z}_{n}\right) \rightarrow L_{q}\left(\mathbb{Z}_{n} \times \mathbb{Z}_{n}\right)$ of the Poisson semigroup $\mathcal{P}_{\psi, t}$. Moreover, if the length $\psi$ on $\mathbb{Z}_{n} \times \mathbb{Z}_{n}$ arises from a (conditionally negative) Poisson-like length function $\varphi$ on $\mathbb{Z}_{n}$ as follows

$$
\psi(k, \ell)=\varphi(k)+\varphi(\ell) \quad \text { for any } \quad 0 \leq k, \ell \leq n-1,
$$

then hypercontractivity estimates for the Poisson semigroup $\mathcal{P}_{\varphi, t}$ associated with $\varphi$ yield similar hypercontractive estimates for $\mathcal{P}_{\psi, t}$. This follows from a general transference result of Bonami, who related, for any compact abelian group $\mathrm{G}$, hypercontractivity in $\mathrm{G}$ and in the infinite cartesian product $\mathrm{G}^{\infty}$ by using Minkowski's inequality [10, Theorem III.2]. In our case, we can transfer $L_{2} \rightarrow L_{q}$ hypercontractive estimates with direct arguments. In fact, we can prove that for any $2 \leq q<\infty$

$$
\left\|\mathcal{P}_{\psi, t}: L_{2}\left(\mathbb{Z}_{n} \times \mathbb{Z}_{n}\right) \rightarrow L_{q}\left(\mathbb{Z}_{n} \times \mathbb{Z}_{n}\right)\right\| \leq\left\|\mathcal{P}_{\varphi, t}: L_{2}\left(\mathbb{Z}_{n}\right) \rightarrow L_{q}\left(\mathbb{Z}_{n}\right)\right\|^{2}
$$

Recall that the Poisson semigroup $\mathcal{P}_{\varphi, t}$ associated with $\varphi$ is given by

$$
\mathcal{P}_{\varphi, t}\left(\widetilde{w}_{k}\right)=e^{-t \varphi(k)} \widetilde{w}_{k}
$$

for any $0 \leq k \leq n-1$, where the family $\widetilde{w}_{k}=e^{\frac{2 \pi i k}{n}}, 0 \leq k \leq n-1$ defines an orthonormal basis of the complex valued function on $\overline{\mathbb{Z}}_{n}$. Let 
$f: \mathbb{Z}_{n} \times \mathbb{Z}_{n} \rightarrow \mathbb{C}$, since $\widetilde{w}_{k, \ell}(a, b)=\widetilde{w}_{k}(a) \widetilde{w}_{\ell}(b)$ we can write

$$
\begin{aligned}
\left\|\mathcal{P}_{\psi, t}(f)\right\|_{L_{q}\left(\mathbb{Z}_{n} \times \mathbb{Z}_{n}\right)} & =\left(\frac{1}{n^{2}} \sum_{a, b=0}^{n-1}\left|\sum_{k, \ell=0}^{n-1} \widehat{f}(k, \ell) e^{-t \psi(k, \ell)} \widetilde{w}_{k, \ell}(a, b)\right|^{q}\right)^{1 / q} \\
& =\left(\frac{1}{n} \sum_{b=0}^{n-1} \frac{1}{n} \sum_{a=0}^{n-1}\left|\sum_{k=0}^{n-1} \widehat{f}_{b}(k) e^{-t \varphi(k)} \widetilde{w}_{k}(a)\right|^{q}\right)^{1 / q} \\
& =\left(\frac{1}{n} \sum_{b=0}^{n-1}\left\|\mathcal{P}_{\varphi, t}\left(f_{b}\right)\right\|_{L_{q}\left(\mathbb{Z}_{n}\right)}^{q}\right)^{1 / q} \\
& \leq\left\|\mathcal{P}_{\varphi, t}\right\|_{2 \rightarrow q}\left(\frac{1}{n} \sum_{b=0}^{n-1}\left\|f_{b}\right\|_{L_{2}\left(\mathbb{Z}_{n}\right)}^{q}\right)^{1 / q}
\end{aligned}
$$

where $f_{b}=\sum_{k=0}^{n-1} \widehat{f}_{b}(k) \widetilde{w}_{k}$ is given by $\widehat{f}_{b}(k)=\sum_{\ell=0}^{n-1} \widehat{f}(k, \ell) e^{-t \varphi(\ell)} \widetilde{w}_{\ell}(b)$. The triangular inequality in $L_{q / 2}\left(\mathbb{Z}_{n}\right)$ then yields

$$
\begin{aligned}
\left\|\mathcal{P}_{\psi, t}(f)\right\|_{L_{q}\left(\mathbb{Z}_{n} \times \mathbb{Z}_{n}\right)} & \leq\left\|\mathcal{P}_{\varphi, t}\right\|_{2 \rightarrow q}\left(\frac{1}{n} \sum_{k=0}^{n-1}\left(\frac{1}{n} \sum_{b=0}^{n-1}\left|\widehat{f}_{b}(k)\right|^{q}\right)^{2 / q}\right)^{1 / 2} \\
& =\left\|\mathcal{P}_{\varphi, t}\right\|_{2 \rightarrow q}\left(\frac{1}{n} \sum_{k=0}^{n-1}\left\|\sum_{\ell=0}^{n-1} \widehat{f}(k, \ell) e^{-t \varphi(\ell)} \widetilde{w}_{\ell}\right\|_{L_{q}\left(\mathbb{Z}_{n}\right)}^{2}\right)^{1 / 2} \\
& \leq\left\|\mathcal{P}_{\varphi, t}\right\|_{2 \rightarrow q}^{2}\|f\|_{L_{2}\left(\mathbb{Z}_{n} \times \mathbb{Z}_{n}\right)},
\end{aligned}
$$

which proves (2.2). In conclusion, whenever the length $\psi$ is of the form (2.1) for some length $\varphi$ on $\mathbb{Z}_{n}$, we are reduced to the hypercontractivity of the Poisson semigroup $\mathcal{P}_{\varphi, t}$ defined on $\mathbb{Z}_{n}$. In particular, a natural Poisson-like length function on $\mathbb{Z}_{n}$ is the word length

$$
\varphi(k)=\min (k, n-k) \quad \text { for any } \quad 0 \leq k \leq n-1 .
$$

Moreover, it is proved in [16, Appendix B] that this length is conditionally negative. By using a combinatorial approach much more involved than the one used to get non-optimal hypercontractive estimates as in (1.1), in conjunction with some numerical estimates, we obtained in $[16$, Theorem A.3] the optimal hypercontractivity time for the Poisson semigroup $\mathcal{P}_{\varphi, t}$ associated with the word length function on $\mathbb{Z}_{n}$. Namely, we established that for any $n \geq 4$

$$
\left\|\mathcal{P}_{\varphi, t}: L_{2}\left(\mathbb{Z}_{n}\right) \rightarrow L_{q}\left(\mathbb{Z}_{n}\right)\right\|=1 \Leftrightarrow t \geq \frac{1}{2} \log (q-1)
$$

whenever one of the following conditions hold

i) $q \in 2 \mathbb{Z}^{+}$and $n$ is even or $n=5$,

ii) $q \in 2 \mathbb{Z}^{+}$and $n \geq 7$ is odd with $n \geq q$.

This result can be regarded as an extension of the two-point inequality which establishes the case $n=2$. The case $n=4$ was then deduced by using a 
reduction argument in [7], whereas the case $n=5$ was proved by Andersson in [3]. We studied in [16] the remaining open cases $n \geq 6$. When $n=3$ the presence of a 3-loop in the Cayley graph prevents from obtaining the expected optimal time, and in fact the optimal time in this case is not even conjectured. This serves to prove Theorem B.

Proof of Theorem B. First observe that the word length $\|$ on $\mathbb{Z}_{n} \times \mathbb{Z}_{n}$ is clearly of the form (2.1) and arises from the word length $\varphi(k)=\min (k, n-k)$ on $\mathbb{Z}_{n}$. Hence || is a c.n.P length. By combining the transference result (2.2) between $\mathcal{P}_{\psi, t}$ and $\mathcal{P}_{\varphi, t}$ with the optimal hypercontractivity for $\mathcal{P}_{\varphi, t}$ stated in (2.3), we obtain that for any $2 \leq q<\infty$

$$
\left\|\mathcal{P}_{||, t}: L_{2}\left(\mathbb{Z}_{n} \times \mathbb{Z}_{n}\right) \rightarrow L_{q}\left(\mathbb{Z}_{n} \times \mathbb{Z}_{n}\right)\right\|=1 \quad \text { for all } \quad t \geq \frac{1}{2} \log (q-1) .
$$

Using (1.2) from the proof of Theorem $\mathrm{A}$, we deduce that for any $q \in 2 \mathbb{Z}_{+}$

$$
\left\|\mathcal{S}_{\mid, t}: L_{2}^{n} \rightarrow L_{q}^{n}\right\|=1 \quad \text { for all } \quad t \geq \frac{1}{2} \log (q-1) .
$$

Moreover, this is optimal by (1.4). Finally, the conditional negativity of || allows us to apply Gross' extrapolation technique as in the proof of Theorem A (ii) with $t_{2,4}=\frac{1}{2} \log 3$ to get $L_{p}^{n} \rightarrow L_{q}^{n}$ hypercontractive estimates.

Acknowledgements. The authors of this paper were supported by the ICMAT Severo Ochoa Grant SEV-2011-0087 (Spain); ERC Starting Grant 256997-CZOSQP (EU); NSF Grant DMS-0901457 (USA); MINECO Grants MTM-2010-16518 \& MTM-2011-26912 (Spain) and the "Ramón y Cajal" program (Spain).

\section{REFERENCES}

[1] Grigori G. Amosov, A remark on the additivity conjecture for a quantum depolarizing channel, Problemy Peredachi Informatsii 42 (2006), no. 2, 3-11.

[2] - On Weyl channels being covariant with respect to the maximum commutative group of unitaries, J. Math. Phys. 48 (2007), no. 1, 012104, 14.

[3] Mats Erik Andersson, Beitrag zur Theorie des Poissonschen Integrals über endlichen Gruppen, Monatsh. Math. 134 (2002), no. 3, 177-190.

[4] Keith Ball, Eric A. Carlen, and Elliott H. Lieb, Sharp uniform convexity and smoothness inequalities for trace norms, Invent. Math. 115 (1994), no. 3, 463-482.

[5] Boaz Barak, Fernando G. S. L. Brandão, Aram W. Harrow, Jonathan Kelner, David Steurer, and Yuan Zhou, Hypercontractivity, sum-of-squares proofs, and their applications [extended abstract], STOC'12 - Proceedings of the 2012 ACM Symposium on Theory of Computing, ACM, New York, 2012, pp. 307-326.

[6] William Beckner, Inequalities in Fourier analysis, Ann. of Math. (2) 102 (1975), no. 1, 159-182.

[7] William Beckner, Svante Janson, and David Jerison, Convolution inequalities on the circle, Conference on harmonic analysis in honor of Antoni Zygmund, Vol. I, II (Chicago, Ill., 1981), Wadsworth Math. Ser., Wadsworth, Belmont, CA, 1983, pp. $32-43$.

[8] J. Bermejo-Vega and M. Van den Nest, Classical simulations of abelian-group normalizer circuits with intermediate measurements, ArXiv 1210.3637, Preprint, 2012. 
[9] Philippe Biane, Free hypercontractivity, Comm. Math. Phys. 184 (1997), no. 2, 457474.

[10] Aline Bonami, Étude des coefficients de Fourier des fonctions de $L^{p}(G)$, Ann. Inst. Fourier (Grenoble) 20 (1970), no. fasc. 2, 335-402 (1971).

[11] Eric A. Carlen and Elliott H. Lieb, Optimal hypercontractivity for Fermi fields and related noncommutative integration inequalities, Comm. Math. Phys. 155 (1993), no. 1, $27-46$.

[12] Toby S. Cubitt, Angelo Lucia, Spyridon Michalakis, and David Pérez-García, Stability of local quantum dissipative systems, arXiv:1303.4744, Preprint, 2013.

[13] Daniel Gottesman, Fault-tolerant quantum computation with higher-dimensional systems, Chaos Solitons Fractals 10 (1999), no. 10, 1749-1758.

[14] Leonard Gross, Logarithmic Sobolev inequalities, Amer. J. Math. 97 (1975), no. 4, 1061-1083.

[15] _ Hypercontractivity, logarithmic Sobolev inequalities, and applications: a survey of surveys, Diffusion, quantum theory, and radically elementary mathematics, Math. Notes, vol. 47, Princeton Univ. Press, Princeton, NJ, 2006, pp. 45-73.

[16] Marius Junge, Carlos Palazuelos, Javier Parcet, and Mathilde Perrin, Hypercontractivity in group von neumann algebras, ArXiv:1304.5789, Preprint, 2012.

[17] Marius Junge, Carlos Palazuelos, Javier Parcet, Mathilde Perrin, and Éric Ricard, Hypercontractivity for free products, arXiv:1211.4759.

[18] Michael J. Kastoryano and Jens Eisert, Rapid mixing implies exponential decay of correlations, J. Math. Phys. 54 (2013), no. 10, 102201, 21.

[19] Michael J. Kastoryano and Kristan Temme, Quantum logarithmic Sobolev inequalities and rapid mixing, J. Math. Phys. 54 (2013), no. 5, 052202, 30.

[20] Subhash A. Khot and Nisheeth K. Vishnoi, The unique games conjecture, integrality gap for cut problems and embeddability of negative type metrics into $\ell_{1}$, Proceedings of the 46th IEEE Symposium on Foundations of Computer Science (FOCS), IEEE, Piscataway, NJ, 2005, pp. 53-62.

[21] Christopher King, Hypercontractivity for semigroups of unital qubit channels, arXiv:1210.8412, Preprint, 2012.

[22] E. Knill, Non-binary unitary error bases and quantum codes, arXiv:quantph/9608048, Preprint, 1996.

[23] Ashley Montanaro, Some applications of hypercontractive inequalities in quantum information theory, J. Math. Phys. 53 (2012), no. 12, 122206, 15.

[24] Ashley Montanaro and Tobias J. Osborne, Quantum Boolean functions, Chic. J. Theoret. Comput. Sci. (2010), Article 1, 41.

[25] Robert Olkiewicz and Bogusław Zegarlinski, Hypercontractivity in noncommutative $L_{p}$ spaces, J. Funct. Anal. 161 (1999), no. 1, 246-285.

[26] Éric Ricard and Quanhua Xu, A noncommutative martingale convexity inequality, ArXiv:1405.0431, Preprint, 2014.

Marius Junge

Department of Mathematics University of Illinois at Urbana-Champaign 1409 W. Green St. Urbana, IL 61891. USA junge@math. uiuc. edu

Carlos Palazuelos Facultad de Ciencias Matemáticas Universidad Complutense de Madrid Plaza de Ciencias s/n, 28040, Madrid. Spain 
Instituto de Ciencias Matemáticas C/ Nicolás Cabrera 13-15. 28049, Madrid. Spain carlospalazuelos@ucm.es

Javier Parcet

Instituto de Ciencias Matemáticas CSIC-UAM-UC3M-UCM

Consejo Superior de Investigaciones Científicas C/ Nicolás Cabrera 13-15. 28049, Madrid. Spain javier.parcet@icmat.es

Mathilde Perrin

Instituto de Ciencias Matemáticas CSIC-UAM-UC3M-UCM

Consejo Superior de Investigaciones Científicas C/ Nicolás Cabrera 13-15. 28049, Madrid. Spain mathilde.perrin@icmat.es 